\title{
In-patient liaison psychiatry in the UK
}

\section{A neglected option for improving the psychiatric care of medical patients}

\author{
David Protheroe and Allan House
}

\begin{abstract}
Aims and method We argue the case for in-patien liaison psychiatry in the UK. We undertook a case note review of the characteristics of a consecutive series of in-patient and day patient admissions to a speciallst liaison psychiatry unit in leeds. We reviewed the literature on in-patient units for the treatment of similar patients, most of which came from the USA.

Reeults Physical morbidity was high in the sample. Forty-four per cent had been admitted for physical problems in the year before admission to the unit. Twenty-three per cent were taking five or more drugs for physical disorders at the time of admission, $85 \%$ were not in paid employment at the time of admission. Treatment involved integrating treatment for physical IIIness, and physical and psychosocial treatments for mental disorder. We identified no reports of in-patient units in the UK doing similar work.

Clinical implications In-patient liaison psychiatry is neglected in the UK to the detriment of a small but important group of patients with coexistent severe physical and mental disorder.
\end{abstract}

Kathol et al (1992) suggest that there are four types of so called medical-psychiatric units in the USA. Type I units provide mainly psychiatric care with a low level of physical illness; Type II units involve psychiatric liaison with the general wards where the main problem is physical illness and the level of psychiatric morbidity is generally low; Type III units provide care for those with moderate physical needs and a wide range in severity of psychiatric illness; and Type IV units can care for those with severe physical and psychiatric illness.

As with any classification, there are likely to be borderline or hybrid cases. For example. Swenson \& Mai (1992) describe the development of a unit in Canada, which comprises six beds on a general hospital psychiatric ward of 49 beds (for a general review of medical-psychiatric units in the USA see Stoudemire \& Fogel (1986) and Fogel \& Stoudemire (1986)).

A further type of in-patient facility is the psychosomatic unit that is particularly a feature of services in Germany. These units treat a different range of illness from their North American counterparts. Physical morbidity is lower and psychosomatic illness is treated with a more psychodynamically-oriented approach (Köhle, 1983; Freyburger et al, 1985; von Rad \& Sellscopp, 1987; Herzog, 1991).

A survey of 11 medical-psychiatric units in the USA by Harsch et al (1991) revealed a marked variation in the types of psychiatric and physical problems treated in different units. Some units acted as tertiary referral centres rather than serving a more local population and some tended to act as specialist centres for treatment of certain syndromes such as chronic pain. Notwithstanding their differences, all the units surveyed were able to provide intravenous therapy, oxygen, and total general nursing care, so these are not simply general psychiatric units under another name, and there were significant differences in patient populations treated by them (Fava et al, 1985).

Specialist in-patient liaison psychiatry units have not been generally developed in the UK. In this paper we describe one British liaison psychiatry in-patient and day patient unit, and discuss the activities of such a unit in the context of UK medical practice.

\section{The study}

The liaison psychiatry unit is situated in the Leeds General Infirmary: a 1000-bed teaching hospital, which does not contain a general psychiatry unit. At the time of this survey, the nursing staff on the unit were doubly qualified, with registered mental nurse (RMN) and registered general nurse (RGN) training. In addition to nursing staff there were occupational therapists, social workers, a clinical psychologist and a behavioural nurse therapist working in the department of liaison psychiatry. The unit had 12 places for adult in-patients and day patients. 
All the in-patient and day patient admissions over a one-year period were surveyed.

\section{Results}

During the 12-month period, 97 patient admissions were recorded. representing 83 patients some being admitted more than once. The 39 men admitted were aged 19-78 years (median $49)$ and the 58 women were aged 18-78 years (median 42). Forty-seven (48\%) of the patients were married or living with a partner, 69 (71\%) were living in some form of family group, and 28 (29\%) were living alone. Only $14(14.5 \%)$ of the patients were in paid employment, full-time or part-time, of whom 11 were working up to the time of admission.

Length of in-patient stay ranged from brief overnight crisis admissions to an admission lasting 209 days. The median length of stay was 17.5 days; $39(40.2 \%)$ patients were admitted as an in-patient for longer than 28 days. Seventeen patients $(17.5 \%)$ attended as daypatients.

\section{Psychiatric and medical history prior to admission}

Forty-nine patients (51\%) had one or more active physical illnesses, proven by investigation, at the time of admission (range 1-3). The range of diagnoses was wide and included unstable insulin-dependent diabetes mellitus, acquired immunodeficiency syndrome (AIDS), chronic renal failure on dialysis, stroke and head injury. As an indication of the severity of these illnesses, 43 patients $(44.3 \%)$ had had at least one urgent non-psychiatric admission within the previous year (range 0-8 admissions). Twenty-six (26.8\%) had one or more routine non-psychiatric admission in the same period (range $0-4$ admissions). On average, patients were taking three prescribed drugs for physical illness at the time of admission (range 0-12), with $22(22.6 \%)$ taking five or more drugs.

Sixty-four patients $(66.0 \%)$ had a previous contact with psychiatry before the liaison admission. Reasons for previous contact included mental disorder in 39 (61\%), unexplained physical symptoms in $14(22 \%)$ and deliberate selfharm in $11(17 \%)$.

\section{Reasons for referral and admission}

The original reason for referral to liaison psychiatry was as follows: coexistent physical illness and mood disorder 39 (40.2\%); physical illness with other comorbid psychiatric disorder 22 (22.6\%); cognitive impairment complicating management $11(11.2 \%)$; unexplained physical symptoms and abnormal illness behaviour 25
(26\%). At the time of admission, the main reason for deciding on admission to the liaison unit rather than general psychiatry was as follows: severity of physical illness 33 (34\%); access to inpatient treatment programme for somatisation 46 (47\%); crisis intervention $11(12 \%)$; patient request seven $(7 \%)$.

\section{Management during the admission}

A team approach is required on the unit, since many of the patients have complex problems. In this sample 32 patients (33.0\%) saw a psychiatric social worker while on the ward, $23(23.7 \%)$ received occupational therapy. $15(15.5 \%)$ received physiotherapy, 11 (11.3\%) saw a dietician and six $(6.2 \%)$ saw a clinical psychologist. For patients with severe physical illness, close liaison is required between psychiatrists and doctors from other specialities, and a system of shared care with a named physician or surgeon has been developed on the unit. Thirty-six of the patients in our care (37.1\%) were treated under shared care for at least part of their stay. Eleven patients (11.3\%) used specialist diagnostic services such as computerised tomography and endoscopy and seven (7.2\%) underwent a surgical intervention during the admission.

Psychiatric treatment was as diverse as the patient group. Just over half received physical treatment for comorbid mental illness. The rest were treated with psychological and social interventions only. The unit employs an eclectic mix of therapeutic approaches. The most frequently used were individual and group therapy. aimed at improving adjustment to illness, occupational therapy (individual and group) aimed at helping patients to maximise function, and individually tailored programmes for the treatment of severe somatising, conversion disorders. and other abnormal illness behaviour.

Of the 93 in-patient episodes, 73 (78.4\%) resulted in discharge home, $13(14.0 \%)$ in discharge to day patient status, two $(2.2 \%)$ in transfer to other psychiatric wards, two (2.2\%) in transfer to medical wards and three (3.2\%) in death.

\section{Discussion}

In terms of the classification described by Kathol et al (1992) the liaison psychiatry unit we have described seems closest to a Type III or Type IV medical-psychiatric unit. It is such units that are most likely to be justified in the National Health Service. Services similar to Kathol's Type I and Type II unit can already be provided by liaison psychiatry services with access to general psychiatry beds, and the psychosomatic approach is not widely accepted in the UK.

The patients admitted to the Leeds unit would not have their psychological needs met in an 
acute medical setting, where lengths of stay are short (average under seven days) and the main focus is on high-dependency care of acute physical illness. Yet they would also be unsuitable for admission to an acute general psychiatry unit where their needs for sustained psychological input may well not be met, and their safety might be jeopardised if they are frail or immobile. In addition, there is the problem that many patients, particularly those with unexplained physical symptoms, are ambivalent about accepting psychiatric treatment. Engagement with psychiatry may be impossible to negotiate if it involves admission to an acute psychiatric ward.

The varied patient mix on the liaison psychiatry unit includes patients with severe physical illness such as AIDS, dialysis-dependent renal fallure or hemiplegic stroke. Acute episodes may require intravenous therapy, and early postoperative care is occasionally necessary. In the same unit there are patients with high levels of disability consequent upon psychological factors. for example patients may be bed-bound with chronic fatigue syndrome or severe unexplained pain or in a wheelchair with hysterical paraplegia. There is a third, smaller group experiencing acute emotional crises, perhaps complicated by recurring self-harm. This case mix is challenging but need not be disadvantageous; common themes emerge during therapy. for example in the overcoming of dependency. finding non-drug approaches to pain, and dealing with the emotional and social impact of illness on others. What it does do is encourage eclecticism; single-theory approaches to typical liaison problems such as pain (Wooley et al. 1978) are difficult to implement and the general tenor is more like that of the unit described by Lipowski (1988) for the in-patient treatment of chronic somatising disorders. It has implications for the recruitment and training and supervision of staff.

Specialist units - particularly in-patient units face constant funding problems and the liaison psychiatry unit described here is no exception. Since this survey was undertaken, the unit has lost its clinical psychology input, and has faced two prolonged periods of bed closure due to underfunding. But what are the alternatives to such a unit? Where one does not exist, then the patients must go somewhere else. Some stay at home, where their families and the primary care services cope with high levels of morbidity and handicap. Those for whom admission becomes inevitable cannot be managed in general psychiatric units, so they end up in acute medical or surgical beds. It is therefore a false economy not to provide this additional and neglected component of care for those severely ill patients who are doubly afflicted by physical and psychiatric disorder.

\section{References}

Fava, G. A., Wise, T. N., Molnar, G., et al (1985) The medical-psychiatric unit: A novel psychosomatic approach. Psychotherapy and Psychosomatics, 43. 194-201.

FOGEl, B. \& STOUdemire, A. (1986) Organization and development of combined medical-psychiatric units: Part 2. Psychosomatics, 27, 417-428.

FReYBURGer, H., KUNSEBECK, H., LEMPA, L., et al (1985) The Hanover consultation liaison model: some empirical findings. Social Science in Medicine, 21, 1391-1401.

HERZOG, T. (1991) In-patient treatment with patients with severe psychosomatic and neurotic disorders: A German perspective. British Joumal of Psychotherapy. 8. 189-198.

HARSCH, H. H., KoRAN, L. M. \& Young, L. D. (1991) A profile of academic medical-psychiatric units. General Hospital Psychiatry. 13, 291-295.

KATHOL, R. G.. HARSCH, H. H., HALL, R. C., et al (1992) Categorization of types of medical/psychiatry units based on level of acuity. Psychosomatics, 3s, 376-386.

KöLе. K. (1983) The psychosomatic ward. Aduances in Psychosomatic Medictne, 11, 176-190.

LiPOWSK, Z. J. (1988) An in-patient programme for persistent somatizers. Canadian Joumal of Psychiatry. 3, 275-278.

von RAD, M. \& SEuschopp, A. (1987) The integrated psychosomatic in-patient unit. A new approach to hospital medical care. Psychotherapy and Psychosomatics, 48, 101-109.

STOUDEMIRE, A. \& FOGEL. B. (1986) Organization and development of combined medical-psychiatric units: Part 1. Psychosomatics, 27, 341-345.

SWEnSON. J. R. \& MA. F. M. (1992) A Canadian medicalpsychiatric in-patient service. Canadian Joumal of Psychiatry. 37, 326-334.

WOOLEY, S., BLACKWEL, B. \& WINGET, C. (1978) A learning theory model of chronic illness behaviour: theory. treatment, and research. Psychosomatic Medicine, 40. $379-401$.

*David Protheroe, Consultant Psychiatrist, Department of Psychiatry. The Northern Hospital, 185 Cooper Street, Epping 3051, Victoria, Australia: and Allan House, Consultant and Senior Lecturer, Department of Liaison Psychiatry, Leeds General Infirmary. Great George Street, Leeds LS1 3EX

*Correspondence 